TIT-HEP-287

YITP/U-95-10

March 1995

\title{
Scaling Behavior of Ricci Curvature at Short Distance near Two Dimensions
}

\author{
Yoshihisa Kitazawa ${ }^{1}$ 円And Masao Ninomiya ${ }^{2}$ ] \\ 1) Department of Physics, Tokyo Institute of Technology, \\ Oh-okayama, Meguro-ku, Tokyo 152, Japan \\ 2) Uji Research Center, Yukawa Institute for Theoretical Physics, \\ Kyoto University, Uji 611, Japan
}

\begin{abstract}
We study the renormalization of the Ricci curvature as an example of generally covariant operators in quantum gravity near two dimensions. We find that it scales with a definite scaling dimension at short distance. The Ricci curvature singularity at the big bang can be viewed as such a scaling phenomenon. The problem of the spacetime singularity may be resolved by the scale invariance of the spacetime at short distance.
\end{abstract}

PACS numbers : 04.60.+n, 98.80Hw

${ }^{*}$ E-mail address : kitazawa@phys.titech.ac.jp

${ }^{\dagger}$ E-mail address : ninomiya@yisun1.yukawa.kyoto-u.ac.jp 
Quantum gravity in $2+\epsilon$ dimensions is capable to describe classical spacetime at long distance and simultaneously a consistent theory at short distance [0] [3]. Therefore it may provide us insights into the short distance structure of our universe. At the short distance fixed point of the renormalization group, the theory becomes scale invariant. Hence the spacetime itself is expected to become scale invariant at short distance. It is very interesting to study the structure of such a scale invariant spacetime.

Recall that the conformal transformation is part of the reparametrization group. Therefore the theory with the reparametrization invariance always possesses the conformal invariance if we regard the conformal mode as a matter field. The rest of the reparametrization group is the volume preserving diffeomorphism. We quantize the conformal mode just like a matter field. In such a quantization scheme, the conformal invariance is a crucial symmetry to respect. However the conformal invariance is known to be broken at the one loop level. In order to resolve this dilemma, we have adopted the tree action which only respects the volume preserving diffeomorphism. Our strategy is to recover the conformal invariance after including the quantum corrections (counter terms). By doing so, the conformal anomaly can be canceled between the tree and the loop contributions [ [,, [5].

It has been shown that this idea is valid at the one loop level and higher order corrections can also be computed [0,6]. The tree level action in $D=2+\epsilon$ dimensions at the short distance fixed point in the renormalization group is

$$
\mu^{\epsilon} / G^{*} \int d^{D} x \sqrt{\hat{g}}\left\{\tilde{R} L\left(\psi, \varphi_{i}\right)--1 / 2 \tilde{g}^{\mu \nu} \partial_{\mu} \psi \partial_{\nu} \psi+1 / 2 \tilde{g}^{\mu \nu} \partial_{\mu} \varphi_{i} \partial_{\nu} \varphi_{i}\right\},
$$

where $L=1+\epsilon /\{8(D-1)\}\left(\psi^{2}-\varphi_{i}^{2}\right)$ and $G^{*}=24 \pi \epsilon /(25-c) . \quad \mu$ is a renormalization scale. In this action $\tilde{g}_{\mu \nu}=\hat{g}_{\mu \rho}\left(e^{h}\right)^{\rho}{ }_{\nu}$ represents the metric without the conformal mode $\psi$. Therefore $h^{\mu}{ }_{\nu}$ is traceless $h^{\mu}{ }_{\mu}=0 . \hat{g}_{\mu \nu}$ is a background metric we have introduced for convenience. Tensor indices are always raised and lowered by the background metric. $\tilde{R}$ is the scalar curvature made out of $\tilde{g}_{\mu \nu} \cdot \varphi_{i}$ denotes $c$ copies of conformally coupled scalar (matter) fields. If $L=\epsilon /\{8(D-1)\}\left(\psi^{2}-\varphi_{i}^{2}\right)$, the action (11) is nothing but the classical Einstein gravity. We have modified the Einstein action minimally to obtain a consistent quantum theory.

The fixed point action possesses the global $Z_{2}$ symmetry: $\psi \rightarrow--\psi$. If the vacuum expectation value of $\psi$ field is nonvanishing, this symmetry is spontaneously broken. Since $\psi$ represents the scale factor of the universe, the vacuum expectation value of $\psi$ is certainly nonvanishing in our universe (in fact it is still growing). In the latter part of this paper, 
we demonstrate this point by investigating the classical solution (15). We therefore identify $\psi$ as the order parameter in quantum gravity. In the weak coupling phase, the vacuum expectation value of $\psi$ is nonvanishing and the $Z_{2}$ invariance is spontaneously broken. Our universe belongs to this phase. In the strong coupling phase, the vacuum expectation value of $\psi$ field vanishes and the global $Z_{2}$ invariance is respected. The short distance fixed point represents the phase transition point between the two.

In order to study the structure of spacetime at short distance, we need to renormalize various operators in the theory. The renormalization of the gauge invariant operators has

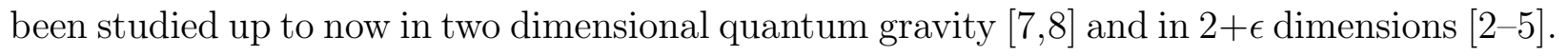
These operators cannot depend on a particular coordinate and are always integrated over the spacetime manifold. The relevant and irrelevant operators are found to acquire large anomalous dimensions of $O(1)$. On the other the anomalous dimensions of the marginal operators are small and of $O(\epsilon)$ at the fixed point.

However we also would like to renormalize generally covariant operators in order to study the structure of spacetime at short distance. The Ricci curvature $R_{\mu \nu}$ is such an example. We would like to know the fate of the spacetime singularity at the beginning of the universe. We may be able to gain insights into such a question by studying the scaling behavior of this operator at short distance. The advantage to consider this operator is that it is invariant under the global conformal transformation. The Riemann curvature $R_{\nu \rho \sigma}^{\mu}$, the Einstein tensor and the energy momentum tensor of matter fields are also of this type. Therefore the anomalous dimension of these operators are expected to be of $O(\epsilon)$. This fact simplifies the renormalization procedure of these operators just like that of the marginal gauge invariant operators.

It has been suggested that we may be able to renormalize generally covariant operators if we relax the general covariance to the covariance under the volume preserving diffeomorphism. We have expected the scaling behavior of these operators [5]. In this paper we show that these expectations are indeed realized at the one loop level.

In quantum theory, the Ricci curvature with the canonical dimension two will acquire the anomalous dimension. Therefore the conformal mode dependence of this operator will be renormalized. We parametrize the metric as $g_{\mu \nu}=\tilde{g}_{\mu \nu} e^{-\phi}$. We separate the conformal mode dependence of $R_{\mu \nu}$ explicitly by the conformal transformation. We reparametrize the conformal mode in terms of $\psi$ which has the canonical kinetic term in the action (1). 
Classically they are related as:

$$
\exp (-\epsilon / 4 \phi)=1+\sqrt{\epsilon /\{8(D-1)\}} \psi
$$

In this parametrization, the Ricci curvature becomes

$$
\begin{aligned}
R_{\mu \nu} & =\tilde{R}_{\mu \nu} \\
& -1 / 2 \tilde{g}_{\mu \nu} \tilde{g}^{\alpha \beta} \nabla_{\alpha} \nabla_{\beta}\left(-\sqrt{2 / \epsilon} \psi+1 / 4 \psi^{2}\right) \\
& -1 / 2 \partial_{\mu} \psi \partial_{\nu} \psi+1 / 2 \tilde{g}_{\mu \nu} \tilde{g}^{\alpha \beta} \partial_{\alpha} \psi \partial_{\beta} \psi+O(\sqrt{\epsilon})
\end{aligned}
$$

where $\tilde{R}_{\mu \nu}$ is the Ricci curvature made out of $\tilde{g}_{\mu \nu}$. The covariant derivative $\nabla_{\mu}$ is taken with respect to $\tilde{g}_{\mu \nu}$.

We have decomposed a generally covariant operator into the collection of the operators which are covariant only with respect to the volume preserving diffeomorphism. These operators need to be subtracted to define renormalized finite operators. As it turns out that the Ricci curvature as a whole cannot be multiplicatively renormalized. In order to circumvent this problem, we introduce different coefficients for different operators and postulate that the renormalized Ricci curvature to be

$$
\begin{aligned}
R_{\mu \nu} & =A \tilde{g}_{\mu \nu} \tilde{g}^{\alpha \beta} \nabla_{\alpha} \nabla_{\beta} \psi--B \tilde{g}_{\mu \nu} \tilde{g}^{\alpha \beta} \nabla_{\alpha} \nabla_{\beta} \psi^{2} \\
& +C \tilde{R}_{\mu \nu}-D \tilde{g}_{\mu \nu} \tilde{R} \\
& -E \partial_{\mu} \psi \partial_{\nu} \psi+F \tilde{g}_{\mu \nu} \tilde{g}^{\alpha \beta} \partial_{\alpha} \psi \partial_{\beta} \psi \\
& +E^{m} \partial_{\mu} \varphi_{i} \partial_{\nu} \varphi_{i}--F^{m} \tilde{g}_{\mu \nu} \tilde{g}^{\alpha \beta} \partial_{\alpha} \varphi_{i} \partial_{\beta} \varphi_{i}
\end{aligned}
$$

In this expression, we have ignored subleading operators in the $\epsilon$ expansion. However we have included the operators which are absent in (2) for the sake of the renormalizability.

As it is shown in the following, the subtractions which are required to make the operators finite close among the operators which are covariant under the volume preserving diffeomorphism. Therefore the Ricci curvature can be renormalized in the form which preserves the covariance under the volume preserving diffeomorphism. The coefficients $\left(A \sim F^{m}\right)$ of the operators can be determined by requiring that the renormalized operator (3) should coincide the classical Ricci curvature (2) at the weak coupling limit, namely at long distance. Since we can derive the renormalization group equations for these coefficients, such calculations can be done at least numerically. However we are most interested in the anomalous dimension of the Ricci curvature at short distance. For such a purpose, it is enough to renormalize the operator around the short distance fixed point and we report the results of such a calculation. 
The one loop renormalization of the operators are performed in a standard background gauge we have utilized in our previous papers. We can ignore the external ghost fields in the background gauge at the one loop level. Firstly we consider the renormalization of the operator $A \tilde{g}_{\mu \nu} \tilde{g}^{\alpha \beta} \nabla_{\alpha} \nabla_{\beta} \psi$. The relevant vertices to renormalize this operator comes only from $\int d^{D} x \sqrt{\hat{g}} 1 / 2 \tilde{g}^{\mu \nu} \partial_{\mu} \psi \partial_{\nu} \psi$ term in the action. We need to consider two one loop diagrams with an external $\psi$ line, to which the operator is inserted.

It is found that this operator can be renormalized multiplicatively at the one loop level. The finite operator with the counter term is

$$
A_{0} \tilde{g}_{\mu \nu} \tilde{g}^{\alpha \beta} \nabla_{\alpha} \nabla_{\beta} \psi=A\left(\tilde{g}_{\mu \nu} \tilde{g}^{\alpha \beta} \nabla_{\alpha} \nabla_{\beta} \psi\right)_{r},
$$

where $A_{0}=(1+g /(2 \pi \epsilon))$ is a bare coefficient and $\left(\tilde{g}_{\mu \nu} \tilde{g}^{\alpha \beta} \nabla_{\alpha} \nabla_{\beta} \psi\right)_{r}$ denotes a renormalized operator.

By demanding the bare coefficient to be independent of the renormalization scale, we find a renormalization group equation for the coefficient $A$ :

$$
\mu d A / d \mu=-G /(2 \pi) A
$$

Therefore $A \sim \mu^{-G / 2 \pi}$ and hence it vanishes at short distance where $\mu \rightarrow \infty$. As it turns out, the renormalization of $B \tilde{g}_{\mu \nu} \tilde{g}^{\alpha \beta} \nabla_{\alpha} \nabla_{\beta} \psi^{2}$ is just analogous and the renormalization group equation for $B$ is

$$
\mu d B / d \mu=-G /(2 \pi) B
$$

Therefore the coefficient $B$ also vanishes at short distance. We suspect that the vanishing of $A$ at short distance may be due to the recovery of $Z_{2}$ symmetry which has been noted in the fixed point action.

Next we move on to renormalize the remaining operators. For this purpose, we need to expand the Ricci curvature $\tilde{R}_{\mu \nu}$ around the background metric in terms of $h_{\mu \nu}$ field up to the quadratic terms. The quadratic terms are

$$
\begin{aligned}
& 1 / 4 \nabla_{\mu} h_{\alpha \beta} \nabla_{\nu} h^{\alpha \beta}--1 / 2 \nabla_{\beta} h_{\alpha \mu} \nabla^{\beta} h^{\alpha}{ }_{\nu}+1 / 2 \nabla^{\beta} h_{\alpha \mu} \nabla^{\alpha} h_{\beta \nu} \\
+ & 1 / 2 \nabla_{\beta}\left(h^{\alpha \beta} \nabla_{\nu} h_{\alpha \mu}\right)+1 / 2 \nabla_{\beta}\left(h^{\alpha \beta} \nabla_{\mu} h_{\alpha \nu}\right)--1 / 2 \nabla_{\beta}\left(h^{\alpha \beta} \nabla_{\alpha} h_{\mu \nu}\right) \\
- & 1 / 4 \nabla_{\alpha} \nabla_{\nu}\left(h^{2}\right)^{\alpha}{ }_{\mu}--1 / 4 \nabla_{\alpha} \nabla_{\mu}\left(h^{2}\right)^{\alpha}{ }_{\nu}+1 / 4 \nabla^{\alpha} \nabla_{\alpha}\left(h^{2}\right)_{\mu \nu} .
\end{aligned}
$$

In this expression, the covariant derivatives are taken with respect to the background metric. There are 13 relevant diagrams for the one loop renormalization. The diagrams constructed 
by using the first three terms of (6) are rather involved to evaluate. In order to simplify this calculation, we utilize the Riemann's normal coordinates [9]. For simplicity the origin of the Riemann's normal coordinate is taken to be that of the propagator. We adopt the doubling trick and complexify $h_{\mu \nu}$ field to further simplify the one loop calculation. The propagator of $h_{\mu \nu}$ field is expanded in terms of the curvature at the origin:

$$
\begin{aligned}
<h^{\alpha \beta}(p) h_{\mu \nu}(-p)> & =G_{0}{ }^{\alpha \beta}{ }_{, \mu \nu} \\
& +1 / p^{2}\left(-1 / 3 \hat{R}+2 / 3 \hat{R}^{\rho \sigma} p_{\rho} p_{\sigma} / p^{2}\right) G_{0}{ }^{\alpha \beta}{ }_{, \mu \nu} \\
& +1 / p^{2}\left(2 \hat{R}_{\rho}^{\alpha}{ }_{\rho}{ }_{\sigma}+2 / D \delta^{\alpha \beta} \hat{R}_{\rho \sigma}\right) G_{0}{ }^{\rho \sigma}{ }_{, \mu \nu}+\cdots,
\end{aligned}
$$

where $G_{0}{ }^{\alpha \beta}{ }_{, \mu \nu}=1 / p^{2}\left(\delta^{\alpha}{ }_{\mu} \delta^{\beta}{ }_{\nu}+\delta^{\alpha}{ }_{\nu} \delta^{\beta}{ }_{\mu}--2 / D \delta^{\alpha \beta} \delta_{\mu \nu}\right)$ is the propagator in the flat spacetime. The second line in the above expression is analogous to that of a free scalar field. With this propagator, the total derivatives of (6) give the one loop divergence of $-G /(2 \pi \epsilon) \tilde{R}_{\mu \nu}$.

In this way, we find the finite operators to be

$$
\begin{aligned}
& C_{0} \tilde{R}_{\mu \nu}-D_{0} \tilde{g}_{\mu \nu} \tilde{R} \\
& -E_{0} \partial_{\mu} \psi \partial_{\nu} \psi+F_{0} \tilde{g}_{\mu \nu} \tilde{g}^{\alpha \beta} \partial_{\alpha} \psi \partial_{\beta} \psi \\
& +E_{0}^{m} \partial_{\mu} \varphi_{i} \partial_{\nu} \varphi_{i}---F_{0}^{m} \tilde{g}_{\mu \nu} \tilde{g}^{\alpha \beta} \partial_{\alpha} \varphi_{i} \partial_{\beta} \varphi_{i} \\
= & C\left(\tilde{R}_{\mu \nu}\right)_{r}-D\left(\tilde{g}_{\mu \nu} R\right)_{r} \\
& -E\left(\partial_{\mu} \psi \partial_{\nu} \psi\right)_{r}+F\left(\tilde{g}_{\mu \nu} \tilde{g}^{\alpha \beta} \partial_{\alpha} \psi \partial_{\beta} \psi\right)_{r} \\
& +E^{m}\left(\partial_{\mu} \varphi_{i} \partial_{\nu} \varphi_{i}\right)_{r}--F^{m}\left(\tilde{g}_{\mu \nu} \tilde{g}^{\alpha \beta} \partial_{\alpha} \varphi_{i} \partial_{\beta} \varphi_{i}\right)_{r} .
\end{aligned}
$$

The bare coefficients are found to be

$$
\begin{aligned}
C_{0} & =C(1-G /(6 \pi \epsilon))+D 4 G /(3 \pi \epsilon)+E G /(12 \pi \epsilon)+E^{m} c G /(12 \pi \epsilon), \\
D_{0} & =D(1+7 G /(6 \pi \epsilon))-C G /(3 \pi \epsilon)+E G /(24 \pi \epsilon)+E^{m} c G /(24 \pi \epsilon), \\
E_{0} & =E-C G /(4 \pi \epsilon) \\
F_{0} & =F(1+G /(2 \pi \epsilon))-E G /(4 \pi \epsilon)--C G /(8 \pi \epsilon), \\
E_{0}^{m} & =E^{m}-C G /(4 \pi \epsilon), \\
F_{0}^{m} & =F^{m}(1+G /(2 \pi \epsilon))-E^{m} G /(4 \pi \epsilon)--C G /(8 \pi \epsilon) .
\end{aligned}
$$

The renormalization group equations follow and from these equations we easily find that

$$
\mu d / d \mu(C-2 D)=-G /(2 \pi)(C-2 D)
$$




$$
\begin{aligned}
\mu d / d \mu(E-2 F) & =-G /(2 \pi)(E-2 F), \\
\mu d / d \mu\left(E^{m}-2 F^{m}\right) & =-G /(2 \pi)\left(E^{m}-2 F^{m}\right) .
\end{aligned}
$$

Therefore at short distance, we can put $C=2 D, E=2 F$ and $E^{m}=2 F^{m}$ in (9) which simplifies the renormalization group equations as

$$
\begin{aligned}
\mu d / d \mu C & =-G /(2 \pi) C--G /(12 \pi) E-c G /(12 \pi) E^{m}, \\
\mu d / d \mu E & =G /(4 \pi) C, \\
\mu d / d \mu E^{m} & =G /(4 \pi) C .
\end{aligned}
$$

The solution of these equations which is symmetric between the conformal mode and matter fields $\left(E=E^{m}\right)$ is found to be

$$
\begin{aligned}
& C=\lambda_{+} \mu^{-\lambda_{+}}+\lambda_{-} \mu^{-\lambda_{-}}, \\
& E=-G /(4 \pi)\left(\mu^{-\lambda_{+}}+\mu^{-\lambda_{-}}\right),
\end{aligned}
$$

where $\lambda_{ \pm}=G /(4 \pi)(1 \pm \sqrt{(2-c) / 3})$. We can show that this is the only nontrivial solution.

The difficulty of this expression is that it becomes complex for $c>2$. However we recall that there is a redundant operator in this channel. Namely the equation of motion with respect $h_{\mu \nu}$ has the same tensor structure with the operator in question. It is a traceless symmetric tensor. Therefore we adopt an interpretation that the operator with the scaling dimension $\lambda_{ \pm}$is redundant.

With this interpretation, the physical renormalization group equations are rather (10). These coefficients show the same scaling behavior with $A$ and $B$. It is also clear that there is no mixing between the physical operators. However they all possess the same scaling exponents. The Einstein tensor does not acquire the anomalous dimension after discarding the redundant operator. It is also the case with the matter energy momentum tensor which is related to the Einstein tensor by the equation of motion. Since the Einstein tensor vanishes identically in two dimensions, these results are reasonable.

Therefore we conclude that the renormalized Ricci curvature scales with the anomalous dimension $-G /(2 \pi)=-12 \epsilon /(25-c)$ at short distance. The renormalization effect is found to soften the short distance singularity.

In order to draw physical implications from the renormalized Ricci curvature let us consider the Robertson-Walker spacetime in $D$ dimensions with the line element:

$$
d s^{2}=-d t^{2}+r(t)^{2} \tilde{g}_{i j} d x^{i} d x^{j}
$$




$$
\tilde{g}_{i j}=\delta_{i j}+k x_{i} x_{j} /\left(1-k|\vec{x}|^{2}\right) .
$$

The Ricci curvature is

$$
\begin{aligned}
& R_{t t}=(D-1) \ddot{r} / r \\
& R_{i j}=-\left(r \ddot{r}+\epsilon(\dot{r})^{2}+\epsilon k\right) \tilde{g}_{i j},
\end{aligned}
$$

where $\dot{r}=d r / d t$.

The cosmological solution of (11) which can be interpreted as the Robertson-Walker spacetime with $k=0$ is

$$
\psi=1 / \sqrt{c} \varphi_{i}=x_{0}, h_{\mu \nu}=0 .
$$

In the classical limit the conformal factor is $\exp (-\phi) \sim x_{0}^{4 / \epsilon}$. By equating the line elements

$$
\begin{aligned}
d s^{2} & =\exp (-\phi)\left(-d x_{0}^{2}+\tilde{g}_{i j} d x^{i} d x^{j}\right) \\
& =-d t^{2}+r^{2} \tilde{g}_{i j} d x^{i} d x^{j},
\end{aligned}
$$

we find $t \sim x_{0}{ }^{D / \epsilon}$ and $r \sim t^{2 / D}$ [5].

Using these relations, we find that the Ricci curvatures $R_{i j}$ and $R_{00}=\left(d t / d x_{0}\right)^{2} R_{t t}$ scales as $x_{0}{ }^{-2}$ in agreement with the canonical dimension. If we extrapolate these classical scaling behaviors at the beginning of the universe where $x_{0} \sim 0$, we encounter the divergence of the Ricci curvature.

Field theoretically we have found that the Ricci curvature scales at short distance. However the scaling exponents have acquired the quantum corrections since the gravitational interaction becomes important at short distance. The main conclusion of this paper is that the scaling dimension of the Ricci curvature has changed from the classical value of 2 to

$$
2-12 \epsilon /(25-c)
$$

to the leading order in the $\epsilon$ expansion at short distance. Although the operator becomes softer in quantum theory, the Ricci curvature still diverges at short distance. We argue that this is not a problem since the theory becomes scale invariant at short distance. In a scale invariant theory, it is natural to expect scaling behaviors of the operators. The Ricci curvature singularity at the big bang can be viewed as a such scaling phenomenon.

Furthermore the quantum gravity in $2+\epsilon$ dimensions is well defined at the short distance limit. In fact we have found that the theory can be extended beyond the big bang and the universe is found to bounce back from the big crunch [5]. In quantum gravity near 
two dimensions, we conclude that the problem of the spacetime singularity is resolved by the scale invariance of the spacetime at short distance. In fact it is a critical phenomena with a universal scaling exponent which is calculable in the $2+\epsilon$ dimensional expansion. Our investigation also suggests the similar mechanism to resolve the spacetime singularity problem at the big bang in our universe.

We are grateful for discussions on this subject to our collaborators, especially T. Aida and H. Kawai. This work is supported in part by the Grant-in-Aid for Scientific Research from the Ministry of Education,Science and Culture. 


\section{References}

[1] S. Weinberg, in General Relativity, an Einstein Centenary Survey, eds. S.W. Hawking and W. Israel (Cambridge University Press, 1979).

R. Gastmans, R. Kallosh and C. Truffin, Nucl. Phys. B133 (1978) 417.

S.M. Christensen and M.J. Duff, Phys. Lett. B79 (1978) 213.

[2] H. Kawai and M. Ninomiya, Nucl. Phys. B336 (1990)115.

[3] H. Kawai, Y. Kitazawa and M. Ninomiya, Nucl. Phys. B393(1993) 280.

[4] H. Kawai, Y. Kitazawa and M. Ninomiya, Nucl. Phys. B404 (1993) 684.

[5] T. Aida, Y. Kitazawa, H. Kawai and M. Ninomiya, Nucl. Phys. B427 (1994) 158.

[6] T. Aida, Y. Kitazawa, J. Nishimura and A. Tsuchiya, KEK-TH-423,TIT-HEP-275,UTKomaba/94-22, to appear in Nucl.Phys. B.

[7] A.M. Polyakov, Mod. Phys. Lett. A2 (1987) 893.

V.G. Knizhnik, A.M. Polyakov and A.B. Zamolodchikov, Mod. Phys. Lett. A3 (1988) 819.

[8] F. David, Mod. Phys. Lett. A3 (1988) 1651.

J. Distler and H. Kawai, Nucl. Phys. B321 (1989) 504.

[9] R. Bailian and C. Bloch, Ann. Phys. 64 (1971) 271. 